\title{
Missing Huntington's Disease for Tardive Dyskinesia: A Preventable Error
}

\author{
Hrishikesh Kumar, Mandar Jog
}

Can. J. Neurol. Sci. 2011; 38: 762-764

Distinguishing spontaneous from drug induced involuntary movements in a patient with psychiatric illness can be a diagnostic challenge to clinicians. Tardive dyskinesia (TD) is a common drug induced movement disorder in such patients but Huntington's disease (HD) also manifests itself with spontaneous abnormal movements and psychiatric abnormalities. ${ }^{1}$ Patients with a psychiatric presentation of undiagnosed HD are often treated with antipsychotics, and on appearance of worsening chorea, many of them could be misdiagnosed as having only TD. Missing the diagnosis of underlying HD has huge clinical, familial and social implications. Over last ten years, 148 cases of HD were diagnosed in our center. Out of them 20 patients were referred as having TD and diagnosis of HD was missed. Casehistory of one such patient is being presented here . Comparison of abnormal movements of these HD patients with the movements in same number of randomly selectd TD patients is provided in Table 1. On the basis of available literature and experience of movement disorders clinics with such patients, we put forward useful clinical criteria to help differentiate between abnormal movements of TD and HD (Table 2).

\section{Case History}

Ms. B, a 46-year-old female, presented with a history of depression and behavioral abnormalities since the age of 22 . She was diagnosed with psychotic depression and received haloperidol along with antidepressants. Her psychiatric symptoms were stable on these medications. At the age of 25, she noticed some abnormal oro-facial and lingual movements. The abnormal movements were attributed to TD related to haloperidol and the drug was subsequently replaced by secondgeneration antipsychotics. The abnormal movements worsened with time and spread to her extremities. Meanwhile she went on to marry and had two children. She was referred to the movement disorders clinic at the age of 42 because of worsening movements. At that time, the patient had generalized chorea with limb predominance, motor impersistence, ataxia, cognitive decline and hyper-reflexia. Because of these features, HD was suspected which was subsequently confirmed by gene testing. She always denied any family history. During a follow up visit, her 72-year-old mother was seen in consultation. She was found to have asymptomatic mild, generalized chorea and motor impersistence. Gene test for HD was positive in her case as well. Ms. B's two sons who were 18 and 16 years old respectively requested the gene test. After proper counseling and informed consent, gene testing was performed and the elder son carried the HD gene mutation.

\section{DiscuSSION}

In this case, the delay between onset of psychiatric symptoms and diagnosis of HD was 20 years. She might have had associated TD but diagnosis of HD was completely missed. In the meantime she had two children, one of whom is gene positive. She did not have the benefit of genetic counseling at the appropriate time. This particular case and other similar patients raise a pertinent question- are there clinical pointers to help community physicians differentiate between the abnormal movements in TD to those in HD? No individual clinical characteristic can be relied upon for the differentiation but taking into account the following clues, one can minimize the chance of error. These clinically helpful clues and their diagnostic value are summarized in Table 2 .

- Stereotypy vs. Chorea: Stereotypy, defined as patterned persistent repetition of same movement, is recognized as an indicator for $\mathrm{TD}^{2}$ On the other hand, chorea with its unpredictability and randomness is considered as important differentiating feature of HD. ${ }^{3}$ But there is considerable overlap - patients with HD can have stereotypy and patients with TD can have choreiform movements. Overlapping clinical features and frequent intake of antipsychotics in HD patients make the differentiation difficult. Obviously, this criterion cannot serve as the sole differentiating feature between TD and HD. Some of the oro-buccal stereotypy, like lip smacking and repetitive tongue protrusion, are more common in patients with TD; if present, they can be a helpful diagnostic marker.

- Suppression of movements by voluntary activities: Amelioration of abnormal movements by action, partial volitional suppressibility and lack of subjective distress are important characteristics in patients with TD that help to differentiate them from HD. ${ }^{4}$ Voluntary activities like speaking and eating reduces the movements in patients with TD but not in HD.

- Speech: Speech is frequently impaired in patients with HD. The abnormalities can include hypophonia, ataxic dysarthria and interruption of speech by oro-bucco-lingual movements. Articulation and speech are relatively preserved in patients of TD

\footnotetext{
From the Movement Disorders Program, London Health Sciences Centre, University of Western Ontario, London, Ontario, Canada.

Received November 18, 2010. Final Revisions Submitted April 29, 2011. Correspondence to: Hrishikesh Kumar, Movement Disorders Program, London Health Sciences Center, University of Western Ontario, London, Ontario, N6A 5A5, Canada.
} 
Table 1: Comparison of abnormal movements of tardive dyskinesia (TD) with that of Huntington's disease (HD)

\begin{tabular}{|c|c|c|}
\hline & TD $(n=20)$ & HD $(n=20)$ \\
\hline \multicolumn{3}{|l|}{ Pattern of involvement } \\
\hline Limb & 4 & 15 \\
\hline Oro-bucco-lingual & 18 & 16 \\
\hline Truncal & 13 & 11 \\
\hline Tongue movement at rest & 17 & 8 \\
\hline Generalized & 2 & 10 \\
\hline Eye movement abnormalities & 0 & 18 \\
\hline \multicolumn{3}{|l|}{ Evolution of movements } \\
\hline Limb onset & 0 & 5 \\
\hline Oro-bucco-lingual onset & 18 & 15 \\
\hline Truncal onset & 2 & 0 \\
\hline \multicolumn{3}{|l|}{ Motor findings } \\
\hline Chorea & 14 & 20 \\
\hline Tremors & 12 & 4 \\
\hline Tics & 6 (vocal- 2, limb 5 , both -1$)$ & 14 (vocal-10, limb-8, both-4) \\
\hline Oro-bucco-lingual stereotypy & 15 & 4 \\
\hline Random oro-bucco- lingual movements & 4 & 12 \\
\hline Lip smacking & 12 & 4 \\
\hline Voluntary suppression of oro-bucco-lingual movements & $13 / 19$ & $0 / 16$ \\
\hline Semipurposiveness of limb movements & $1 / 4$ & $12 / 15$ \\
\hline Akathisia & 4 & 1 \\
\hline Dystonia & $8($ Axial $6, \operatorname{limb} 3$, both 1$)$ & 2 (hand and foot dystonia in both) \\
\hline Limb ataxia & 0 & 8 \\
\hline Spasticity & 0 & 4 \\
\hline Parkinsonism & 8 & 4 \\
\hline Tongue impersistence & 0 & 13 \\
\hline Oculomotor impersistence & 0 & 15 \\
\hline Grip impersistence & 0 & 7 \\
\hline Balance and gait problems & 1 & 16 \\
\hline Articulation and speech abnormality & 2 & 11 \\
\hline
\end{tabular}

even in the presence of significant oro-bucco-lingual movements.

- Pattern of body part involvement: Oro-bucco-lingual movements are often first to appear in patients in both the groups, but they are more common in patients with TD. Truncal involvement is much more common than limb involvement in TD. In contrast, limb movements are commoner than truncal movements in HD. Presence of generalized abnormal movements indicates HD rather than TD. As in oro-buccolingual movements, limb movements are partially suppressible and result in limited or no functional impairment in TD. ${ }^{4}$

- Semi-purposiveness of abnormal movements: Incorporation of involuntary limb movements into some voluntary activities (semi-purposiveness) is frequently seen in patients with HD but is rare in TD. ${ }^{1-3}$ Semi-purposeful movement has been included in the definition of chorea in classical literature. Lack of this phenomenon in the patients with TD might suggest that abnormal movements in TD are 'chorea like movements' and not true chorea.

- Eye movement abnormalities: Eye movement abnormalities can appear early in HD..$^{5}$ They include slow initiation of saccades, saccadic intrusions of pursuits, oculomotor impersistence and impaired optokinetic nystagmus (OKN). Eye movement abnormalities are generally not seen in patients with TD. A careful eye movement examination has immense value in differentiation between TD and HD.

- Motor impersistence: All the patients with psychiatric features and abnormal movements should be examined for motor impersistence. Oculomotor, tongue and grip impersistence are not universal finding but if present, they are highly suggestive of HD. Among them, oculomotor impersistence is the most sensitive sign (Table 1). For examination of impersistence, patients are asked to maintain sustained muscular activity of corresponding body part (looking towards one side, protrusion of tongue without touching the teeth or lips, maintaining a tight hand-grip around examiner's finger) for 30 seconds. In cases of impersistence, the eye ball will frequently tend to come to midline, tongue will move in and out (Jack in box sign) and hand-grip will fluctuate (milk-maid grip).

- Additional neurological signs: Retrocollis, opisthotonus and axial dystonia favor the diagnosis of TD, whereas presence of ataxia, gait imbalance, spasticity, frontal lobe signs and aphasia favors HD. ${ }^{1-3}$

Huntington's disease can be misdiagnosed as TD because of presence of psychiatric symptoms, history of antipsychotic intake, abnormal hyperkinetic movements and prominent oro- 
Table 2: Clinical differentiation between abnormal movement of tardive dyskinesia (TD) and Huntington's Disease (HD)

\begin{tabular}{|c|c|c|}
\hline & TD & HD \\
\hline Oro-bucco-lingual movements & $\begin{array}{lll}\begin{array}{l}\text { Stereotypy } \\
\text { movements }\end{array} & > & \text { Random } \\
\end{array}$ & Random movements $>>$ Stereotypy \\
\hline Lip smacking and tongue protrusion & ++ & $+/-$ \\
\hline Tongue movement at rest & +++ & + \\
\hline Voluntary suppression of oro-buccal movements & +++ & $+/-$ \\
\hline Speech and language & Less commonly affected & affected \\
\hline Pattern of movements & truncal $\gg>\operatorname{limb}$ & limb $\gg>$ truncal \\
\hline Generalized movement & + & ++ \\
\hline Semipurposiveness of limb movement & $+/-$ & +++ \\
\hline Balance and gait & Not affected by movements & Affected by movements. \\
\hline Akathisia & + & $+/-$ \\
\hline Eye movement abnormalities & - & +++ \\
\hline Motor impersistence & - & +++ \\
\hline Parkinsonism & ++ & + \\
\hline Axial dystonia (retrocollis, Opisthotonous) & ++ & $+/-$ \\
\hline Tics & + & + \\
\hline Ataxia & - & ++ \\
\hline Spasticity & - & ++ \\
\hline Frontal lobe signs & - & ++ \\
\hline
\end{tabular}

buccal involvement. On the other hand a patient with HD can have co-existent TD and the diagnosis of HD can be completely missed. Careful observation of abnormal movements can help in differentiating these two conditions with a reasonable degree of accuracy. Repetitive character, partial voluntary suppressibility, amelioration by action, limited functional impairment, truncal predominance of movements, retrocollis, and opisthotonus suggest the possibility of TD. Randomness and inability to suppress the movements, involvement of speech, language and gait, semipurposive nature of the limb movements, motor impersistence, eye movement abnormalities, frontal lobe signs and presence of ataxia or spasticity favor the diagnosis of HD. Clinical differentiation can guide community physicians in selecting a patient for genetic testing.

\section{REFERENCES}

1. Marsden CD, Tarsy D, Baldessarini RJ. Spontaneous and drug induced movement disorders in psychotic patients. In: Benson DF, Blumer D, editors. Psychiatric aspect of neurological disease. New York: Grune and Statton; 1975. p. 219-65.

2. Cummings JL, Wirshing WC. Recognition and differential diagnosis of tardive dyskinesia. Int J Psychiatry Med. 1989;19 (2): $133-44$

3. Wild EJ, Tabrizi SJ. The differential diagnosis of chorea. Pract Neurol. 2007;7(6): 360-73.

4. Walters AS, McHale D, Sage JI, Hening WA, Bergen M. A blinded study of the suppressibility of involuntary movements in Huntington's chorea, tardive dyskinesia, and L-dopa-induced chorea. Clin Neuropharmacol. 1990;13(3):236-40.

5. Leigh RJ, Newman SA, Folstein SE, Lasker AG, Jensen BA. Abnormal ocular motor control in Huntington's disease. Neurology. 1983;33(10):1268-75. 